\title{
Effects of Variable Fluid Properties and MHD on Mixed Convection Heat Transfer from a Vertical Heated Plate Embedded in a Sparsely Packed Porous Medium
}

\author{
${ }^{1}$ Nalinakshi N, ${ }^{2}$ Dinesh P. A. and ${ }^{3}$ Chandrashekar D. V \\ ${ }^{1}$ Department of Mathematics, Atria Institute of Technology, Bangalore-560 024, INDIA \\ ${ }^{2}$ Department of Mathematics, M. S. Ramaiah Institute of Technology, Bangalore - 560 054, INDIA \\ ${ }^{3}$ Department of Mathematics, Vivekananda Institute of Technology, Bangalore - 560 074, INDIA
}

\begin{abstract}
The effects of variable Fluid Properties like variation of permeability, porosity, thermal conductivity and magnetic field on Mixed Convection Heat transfer from Vertical Heated Plate Embedded in a Sparsely Packed Porous Medium have been approached numerically. The boundary layer flow in the porous medium is governed by Lapwood - Forchheimer - Brinkman extended Darcy model and the Lorentz force. The natures of these equations are highly non-linear and coupled each other. The non-linear differential equations are non-dimensionalised using the non-dimensional parameter involving Grashoff number Gr, Prandtl number Pr, Hartmann number M, Eckert number $E$ and so on. Similarity transformations are employed and the resulting ordinary differential equations are solved numerically by using shooting algorithm with Runge - Kutta and Newton - Raphson method to obtain velocity and temperature distributions. Besides, skin friction and Nusselt number are also computed for various physical parameters governing the problem under consideration. It is found that the inertial parameter has a significant influence in decreasing the flow field, whereas its influence is reversed on the rate of heat transfer for all values of permeability considered. The effect of Magnetic field is diminution with velocity of the fluid flow. Further, the obtained results under the two limiting conditions were found to be in good agreement with the existing results. Keywords: Heat transfer, MHD, Newtonian fluid, porous medium, similarity solution.
\end{abstract}

\section{Introduction}

For past decades, flow through porous medium has received attention of many researches because of its extensive applications in chemical engineering, enhanced recovery of petroleum resources, packed bed reactors etc. The study of mixed convection (free and forced convection) in porous medium has also been given much attention in the recent years. Considerable attention has been evinced on the study of boundary layer flow behavior and heat transfer characteristics of Newtonian fluid past and/or through a vertical plate embedded in a fluid saturated porous medium because of its wide spectrum of applications in engineering processes. Many Investigators have obtained similarity solutions for the problem of free convection heat transfer from a vertical plate embedded in a fluid saturated porous medium. Merkin[1] gave a similarity analysis of a combined free and forced convection flow past an isothermal semi-infinite vertical plate. The combined free and forced convection from vertical plates in porous media has been investigated by Ranganathan and Viskanta[2]. All the above mentioned studies treat the permeability and conductivity or thermal resistance of the medium as constants. Hsieh et al[3] have obtained nonsimilar solution for combined convection from vertical plates in porous media with variable surface temperature or heat flux.

The convection of liquid metal under the influence of a magnetic field has been studied extensively. Pioneering studies on the effect of a magnetic field on the convection of electro-conducting materials were basically carried out in the fields of the geophysics and cosmology and were related to convection of the Earth's mantle or of gas in a space which was analyzed by Saltzmann [4]. Aldoss et al [5] have studied mixed convection from a vertical plate embedded in a porous medium in the presence of magnetic field. Several investigators have studied the effect of the magnetic field on the free convection flow over a semi-infinite vertical plate. Nield and Bejan[6] studied Darcy flow in an isotropic medium with the effect of a magnetic field and the flow in a medium with anisotropic permeability. He found the effect of uniform applied magnetic field is to reduce the effective permeability in the case of boundary layer flow. Elbashbeshy[7] investigated Heat and mass transfer along a vertical plate with variable temperature and concentration in the presence of magnetic field.

In some industrial applications, such as fixed-bed catalytic reactors, packed bed heat exchangers and drying, the value of the porosity is maximum at the wall and minimum away from the wall so the porosity of the porous medium should be taken as non - uniform. Porosity measurements by Shwartz and Smith[8] and Benenati and Brosilow [9] show that porosity is not constant but varies from the wall to the interior of the porous medium due to which permeability also varies. Chandrasekhara et al[10] has incorporated the variable permeability to study the flow past and through a porous medium and have shown that the variation of porosity and permeability has greater 
influence on velocity distribution and on heat transfer. On the other hand, Chandrasekhar and Namboodiri [11] have shown the effectiveness of variable permeability of the porous medium on velocity distribution and heat transfer. Nevertheless, the inertia effects become important in a sparsely packed porous medium and hence their effect on mixed convection problems needs to be investigated. Mohammadein and El-shaer[12] studied mixed convective flow past a semi-infinite vertical plate embedded in a porous medium incorporating the variable permeability in Darcy's model. Sri Hari Babu and Ramana Reddy [13] discussed the effects of mass transfer on MHD mixed convection flow past an infinite vertical plate with ohmic heating and viscous dissipation. Mahinder Singh et al [14]studied by considering the effect of uniform vertical magnetic field and uniform vertical rotation on thermosolutal convection in a layer of an electrically conducting couple-stress fluid heated and soluted from below. Recently, Pal and Shivakumar[15] analyzed mixed convection heat transfer from a vertical heated plate embedded in a Newtonian fluid sparsely packed porous medium by considering the variation of permeability, porosity and thermal conductivity. Dulal Pal[16] studied magneto hydrodynamic non-Darcy mixed convection heat transfer from a vertical heated plate embedded in a porous medium with variable porosity, by taking the viscous dissipation term in the energy equation. Pal[17] also made analysis of mixed convection heat transfer in the boundary layers on an exponentially stretching surface with magnetic field. Nalinakshi et al[18] found numerical solutions for heat transfer from a vertical heated plate embedded in a Newtonian fluid sparsely packed porous medium considering the variable fluid properties with the influence of inertial parameter.

The aim of the present investigation is therefore, to study systematically and numerically the effect of inertial terms on combined free and forced convective heat transfer past a semi-infinite vertical plate embedded in a saturated porous medium in the presence of magnetic field with variable permeability, porosity and thermal conductivity. In this analysis coupled non-linear partial differential equations, governing the problem, are first reduced to the ordinary differential equations by similarity transformations and then the resultant boundary value problem is converted into the system of five simultaneous equations of first-order for five unknowns. These equations are then solved numerically to obtain velocity distributions and temperature profiles for various values of physical parameters. The results obtained are compared in the absence of magnetic field with the numerical computation under limiting conditions agree well with the existing ones and thus verifies the accuracy of the method used.

\section{Mathematical Formulation}

We consider two-dimensional steady flow of a laminar, incompressible, viscous, electrically conducting fluid past a semi-infinite vertical heated plate embedded in a sparsely packed Newtonian fluid saturated porous medium of variable porosity, permeability and thermal conductivity. A uniform transverse magnetic field is applied to the plate. The flow is assumed to be dominated by viscous, Lorentz and pressure forces with inertia. The plate is assumed to be electrically non-conducting, as many fluids used in the laboratory, the conductivity is usually small. The $\mathrm{x}$-coordinate is measured along the plate from its leading edge, and y-coordinate normal to it (see fig.1). Let $\mathrm{U}_{0}$ be the velocity of the fluid in the upward direction and the gravitational field, $\mathrm{g}$, is acting in the downward direction. The plate is maintained at a uniform temperature $\mathrm{T}_{\mathrm{w}}$ which is always greater than the free stream values existing far from the plate (i.e. $T_{w}>T_{\infty}$ ).

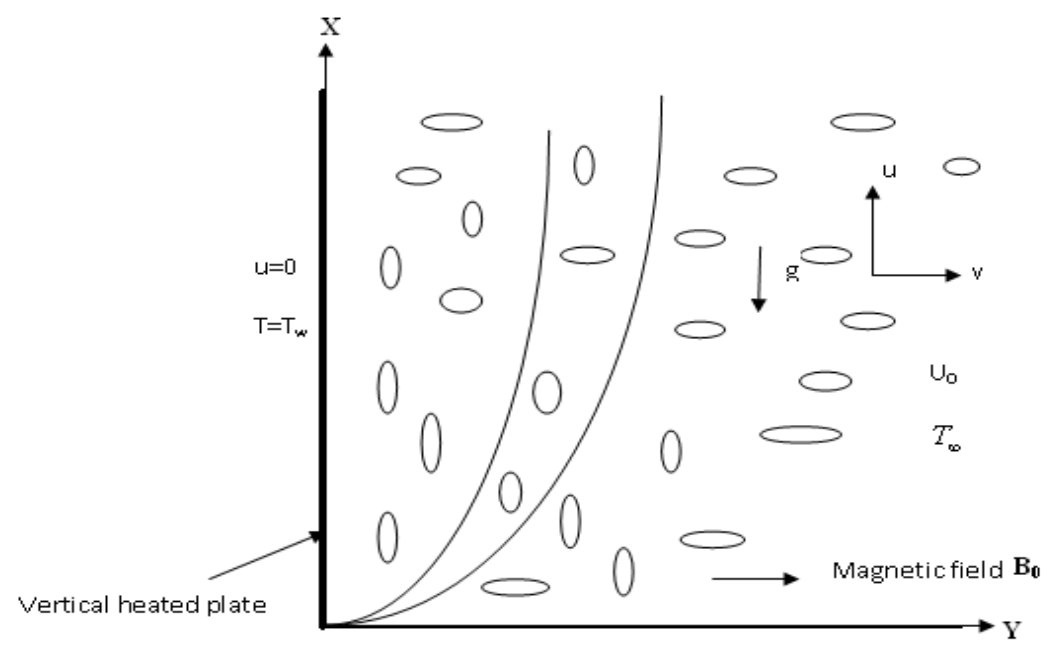

Fig.1. Physical configuration of the problem

The boundary layer equations governing the conservation law of mass, momentum and energy (heat) for incompressible steady viscous and electrically conducting flow can be written in the following form: 


$$
\begin{aligned}
& \frac{\partial u}{\partial x}+\frac{\partial v}{\partial y}=0 \\
& u \frac{\partial u}{\partial x}+v \frac{\partial v}{\partial y}=g \beta\left(T-T_{\infty}\right)+\frac{\bar{\mu}}{\rho} \frac{\partial^{2} u}{\partial y^{2}}+\frac{\mu}{\rho} \frac{\varepsilon(y)}{k(y)}\left(U_{0}-u\right)+F \frac{\varepsilon^{2}(y)}{\sqrt{k(y)}}\left(U_{0}^{2}-u^{2}\right)+\frac{\sigma_{m} B_{0}^{2}}{\rho} \varepsilon(y)\left(U_{0}-u\right)(2) \\
& \left(u \frac{\partial T}{\partial x}+v \frac{\partial T}{\partial y}\right)=\frac{\partial}{\partial y}\left(\alpha(y) \frac{\partial T}{\partial y}\right)+\frac{\bar{\mu}}{\rho C_{p}}\left(\frac{\partial u}{\partial y}\right)^{2}
\end{aligned}
$$

where $\mathrm{u}$ and $\mathrm{v}$ are the velocity components along the $\mathrm{x}$ and $\mathrm{y}$ direction, respectively, $\mathrm{T}$ is the temperature of the fluid, $\rho$ is the fluid density, $\bar{\mu}$ is the effective viscosity of the fluid, $\mu$ is the fluid viscosity, $\mathrm{k}(\mathrm{y})$ is the variable permeability of the porous medium, $\varepsilon(y)$ is the porosity of the saturated porous medium, $\alpha(y)$ is the variable effective thermal diffusivity of the medium, $\mathrm{F}$ is the empirical constant of the second -order resistance term due to inertial effect, $\mathrm{C}_{\mathrm{p}}$ is the specific heat at constant pressure, $\beta$ is the coefficient of volume expansion. $B_{0}$ is the applied magnetic field, $\sigma_{m}$ is the electrical conductivity.

The above governing equations need to be solved subject to the following boundary conditions on velocity and temperature fields:

$$
\begin{array}{lll}
u=0, & v=0, \quad T=T_{w} & \text { at } y=0 \\
u=U_{o}, & v=0, \quad T=T_{\infty} & \text { as } y \rightarrow \infty
\end{array}
$$

Since the flow field is uniform at a sufficiently large distance from the porous surface, so in the free stream $u=U_{0}$, where $U_{0}$ is the free stream velocity and $T_{\infty}$ is the ambient temperature.

We now introduce the following dimensionless variables $f$ and $\theta$ as well as the similarity variable $\eta$ - see Mohammadein and El-shaer [12]:

$\eta=\left(\frac{y}{x}\right)\left(\frac{U_{0} x}{v}\right)^{1 / 2}, \quad \psi=\sqrt{v \mathrm{U}_{0} x} \mathrm{f}(\eta), \theta=\frac{\mathrm{T}-\mathrm{T}_{\infty}}{\mathrm{T}_{\mathrm{w}}-T_{\infty}}$

$\psi(x, y)$ is the stream function defined by $u=\frac{\partial \psi}{\partial y}, \mathrm{v}=-\frac{\partial \psi}{\partial x}$, such that the continuity equation (1) is satisfied,

$\mathrm{T}_{\mathrm{w}}$ is the plate tempeature and the velocity components is given by

$u=U_{o} f^{\prime}(\eta), \quad v=-\frac{1}{2} \sqrt{\frac{v U_{o}}{x}}\left(f(\eta)-\eta f^{\prime}(\eta)\right)$

Where a prime represents differentiation with respect to $\eta$.

Here we consider the variable permeability $k(\eta)$, variable porosity $\varepsilon(\eta)$ and variable thermal conductivity $\alpha(\eta)$ to decrease exponentially with the normal distance to the wall, from a value close to one at the solid boundaries to $\mathrm{k}_{\mathrm{o}}, \varepsilon_{\mathrm{o}}$ and $\alpha_{0}$ value at the edge of the boundary layer [see Chandrasekhara and Namboodiri(1985)].

$$
\begin{aligned}
& k(\eta)=k_{0}\left(1+d e^{-\eta}\right) \\
& \varepsilon(\eta)=\varepsilon_{0}\left(1+d^{*} e^{-\eta}\right) \\
& \alpha(\eta)=\alpha_{0}\left[\varepsilon_{0}\left(1+d^{*} e^{-\eta}\right)+\sigma^{*}\left\{1-\varepsilon_{0}\left(1+d^{*} e^{-\eta}\right)\right\}\right]
\end{aligned}
$$

Where $\mathrm{k}_{\mathrm{o}}, \varepsilon_{\mathrm{o}}$ and $\alpha_{0}$ are the permeability, porosity and conductivity respectively at the edge of the boundary

layer, respectively. $\mathrm{d}$ and $\mathrm{d}^{*}$ are treated as constants having values 3.0 and 1.5 respectively, for variable permeability (VP)and $d=d^{*}=0$ for uniform permeability (UP). where $\sigma^{*}$ is the ratio of the thermal conductivity of solid to the conductivity of the fluid.

Substituting (6) and (7) in Equations (2) and (3), we get the following transformed equations: 


$$
\begin{aligned}
& f^{\prime \prime \prime}+\frac{1}{2} f f^{\prime \prime}+\frac{G r}{\operatorname{Re}^{2}} \theta+\frac{\alpha^{*}\left(1+d^{*} e^{-\eta}\right)}{\sigma \operatorname{Re}\left(1+\mathrm{de}^{-\eta}\right)}\left(1-f^{\prime}\right)+\frac{\beta^{*}\left(1+d^{*} e^{-\eta}\right)}{\left(1+d \mathrm{e}^{-\eta}\right)^{1 / 2}}\left(1-f^{\prime 2}\right)+M^{2} \varepsilon_{0}\left(1+d^{*} e^{-\eta}\right)\left(1-f^{\prime}\right)=0 \\
& \theta^{\prime \prime}=-\frac{(1 / 2) \operatorname{Pr} \theta^{\prime} f+\operatorname{Pr} E f^{\prime \prime 2}+\varepsilon_{0} d^{*} e^{-\eta}\left(\sigma^{*}-1\right) \theta^{\prime}}{\varepsilon_{0}+\sigma^{*}\left(1-\varepsilon_{0}\right)+\varepsilon_{0} d^{*} e^{-\eta}\left(1-\sigma^{*}\right)}
\end{aligned}
$$

where, $\beta^{*}=F \varepsilon_{o}^{2} x / k_{o}^{1 / 2}$ is the local inertial parameter, $\operatorname{Pr}=\bar{\mu} / \rho \alpha_{o}$ is the Prandtl number, $\alpha^{*}=\mu / \bar{\mu}$ is the ratio of viscosities, $E=U_{o}^{2} / C_{p}\left(T_{w}-T_{\infty}\right)$ is the Eckert number, $\sigma=k_{o} / x^{2} \varepsilon_{o}$ is the local permeability parameter, $\operatorname{Re}=U_{o} x / v$ is the local Reynolds number and $G r=g \beta\left(T_{w}-T_{\infty}\right) x^{3} / v^{2}$ is the local Grashof number and $M^{2}=\frac{\sigma_{m} B_{0}^{2} x}{\left(\rho U_{0}\right)}$ is the local magnetic field parameter.

The transformed boundary conditions are:

$$
\begin{aligned}
& f=0, f^{\prime}=0, \theta=1 \quad \text { at } \eta=0 \\
& f^{\prime}=1, \theta=0 \quad \text { as } \eta \rightarrow \infty
\end{aligned}
$$

Once the velocity and temperature distributions are known, the skin friction and the rate of heat transfer can be calculated respectively by

$$
\begin{aligned}
& \tau=-f^{\prime \prime}(0) / \sqrt{\operatorname{Re}} \\
& N u=-\sqrt{\operatorname{Re}} \theta^{\prime}(0)
\end{aligned}
$$

where $\tau$ is the skin friction and $\mathrm{Nu}$ is the Nusselt number.

\section{Numerical Method}

Equations (11) and (12) constitute a highly non-linear coupled boundary value problem of third and second order respectively. An improved numerical scheme involving shooting technique with Runge-Kutta-Fehlberg method is developed to solve the resulting nonlinear boundary value problem. Thus, the coupled nonlinear boundary value problem of third-order in $\mathrm{f}$ and second-order in $\theta$ has been reduced to a system of five simultaneous equations of first-order for five unknowns as follows (see vajravelu[19]):

$$
\begin{aligned}
& f_{1}^{\prime}=f_{2}, \\
& f_{2}^{\prime}=f_{3}, \\
& f_{3}^{\prime}=-\frac{1}{2} f_{1} f_{3}-\frac{G r}{\operatorname{Re}^{2}} f_{4}-\left(\frac{\alpha^{*}}{\sigma \operatorname{Re}}\right) \frac{1+d^{*} e^{-\eta}}{1+d \mathrm{e}^{-\eta}}\left(1-f_{2}\right)-\frac{\beta^{*}\left(1+d^{*} e^{-\eta}\right)}{\left(1+d \mathrm{e}^{-\eta}\right)^{1 / 2}}\left(1-f_{2}^{2}\right)-M^{2} \varepsilon_{0}\left(1+d^{*} e^{-\eta}\right)\left(1-f_{2}\right) \\
& f_{4}^{\prime}=f_{5}, \\
& f_{5}^{\prime}=-\frac{(1 / 2) \operatorname{Pr} f_{1} f_{5}+\operatorname{Pr} E f_{3}^{2}+\varepsilon_{0} d^{*} e^{-\eta}\left(\sigma^{*}-1\right) f_{5}}{\varepsilon_{0}+\sigma^{*}\left(1-\varepsilon_{0}\right)+\varepsilon_{0} d^{*} e^{-\eta}\left(1-\sigma^{*}\right)}
\end{aligned}
$$

where $f_{1}=f, f_{2}=f^{\prime}, f_{3}=f^{\prime \prime}, f_{4}=\theta, f_{5}=\theta^{\prime}$ and a prime denotes differentiation with respect to $\eta$ The boundary conditions now become

$$
\begin{array}{ll}
f_{1}=0, \mathrm{f}_{2}=0, \mathrm{f}_{4}=1 & \text { at } \eta=0 \\
f_{2}=1, \quad \mathrm{f}_{4}=0 & \text { as } \eta \rightarrow \infty
\end{array}
$$

To solve the system of first-order differential equations along with boundary conditions, we need five initial conditions, but we have only two initial condition on $f$ and one initial condition on $\theta$. The third condition on $f$ $\left(i . e . f^{\prime \prime}(0)\right)$ and second condition on $\theta\left(i . e . \theta^{\prime}(0)\right)$ are not prescribed, which are determined by employing numerical shooting method and using the two ending boundary condition given in Eq.(19). The selection of an appropriate finite value of $\eta_{\infty}$ to be made. A good guess of the initial condition in the shooting technique is to be made on which the convergence depends. The iterative process is terminated when the difference between two successive values reached $10^{-6}$, then the solution is said to have converged results. The slight deviation in the values may be due to the 
use of Runge-Kutta-Fehlberg method which has fifth order accuracy whereas, Mohammadein and El-Shaer[12] have used fourth-order Runge-Kutta method which has only fourth order accuracy. Thus the present results are more accurate compared to their results.

\section{Results and Discussion}

The system of first-order differential equation (17) - (19) is solved numerically using shooting technique with Runge-kutta-Fehlberg method. In order to know the accuracy of the method used, computed values of $f^{\prime \prime}(0)$ and $\theta^{\prime}(0)$ were obtained for $\beta^{*}=0, \quad \mathrm{M}=0$ and compared with those obtained by Mohammadein and El-shaer (2004) in Table 1 for the variable permeability $\left(\mathrm{d}=3.0, \mathrm{~d}^{*}=1.5\right)$ case and good agreement has been obtained with their results. The values tabulated in Table 1 for $\varepsilon_{0}=0.4, \mathrm{E}=0.1, \operatorname{Pr}=0.71 \& 7.0$ with selected values of $\mathrm{Gr} / \operatorname{Re}^{2}, \sigma^{*}$ and $\alpha^{*} / \sigma \operatorname{Re}$. Table 2 contain the computed values of $f^{\prime \prime}(0)$ and $-\theta^{\prime}(0)$ for the selected values of $\alpha^{*} / \sigma \operatorname{Re}, \mathrm{Gr} / \operatorname{Re}^{2}, \mathrm{M}, \sigma^{*}$ and $\beta^{*}$, for uniform permeability (UP) and variable permeability (VP) cases, it is observed that an increase in the value of $\beta^{*}$ is to increase the skin friction for all the values of $\alpha^{*} / \sigma \operatorname{Re}$ and $\mathrm{Gr} / \mathrm{Re}^{2}$ in the case of both UP and VP. Further, it is interesting to note that the effect of $\sigma^{*}$ is to increase the skin friction. Also from Table 2 it is analyzed that increase in the value of $\beta^{*}$ is to decrease the rate of heat transfer for lower value of $\mathrm{Gr} / \mathrm{Re}^{2}$ for both UP and VP but reverse trend is seen for higher values of $G r / \mathrm{Re}^{2}$. Further, it is noted that the effect of $\sigma^{*}$ is to increase the rate of heat transfer for UP whereas reverse trend is seen in the case of VP.

Table 1: Results for $f^{\prime \prime}(0)$ and $-\theta^{\prime}(0)$ for $\operatorname{Pr}=0.71$ and $7.0, \beta^{*}=0.0$ for variable permeability case

\begin{tabular}{|c|c|c|c|c|c|c|c|c|c|c|c|}
\hline \multirow[t]{3}{*}{$\bar{M}$} & \multirow{3}{*}{$\sigma^{*}$} & \multirow{3}{*}{$\frac{G r}{\operatorname{Re}^{2}}$} & \multirow{3}{*}{$\frac{\alpha^{*}}{\sigma \operatorname{Re}}$} & \multicolumn{4}{|c|}{ Present value } & \multicolumn{4}{|c|}{ Mohammadein and El-shaer } \\
\hline & & & & \multicolumn{2}{|l|}{$\operatorname{Pr}=0.71$} & \multicolumn{2}{|l|}{$\operatorname{Pr}=7.0$} & \multicolumn{2}{|l|}{$\operatorname{Pr}=0.71$} & \multicolumn{2}{|l|}{$\operatorname{Pr}=7.0$} \\
\hline & & & & $f^{\prime \prime}(0)$ & $-\theta^{\prime}(0)$ & $f^{\prime \prime}(0)$ & $-\theta^{\prime}(0)$ & $f^{\prime \prime}(0)$ & $-\theta^{\prime}(0$ & $f^{\prime \prime}(0)$ & $-\theta^{\prime}(0)$ \\
\hline \multirow[t]{18}{*}{0.0} & \multirow{9}{*}{$\begin{array}{l}2 . \\
0\end{array}$} & 0.2 & 0.0 & 0.61304 & 0.38234 & 0.495610 & 0.69497 & 0.61215 & 0.38030 & 0.49631 & 0.70036 \\
\hline & & & 0.1 & 0.64626 & 0.38456 & 0.541172 & 0.69985 & 0.64526 & 0.38281 & 0.54207 & 0.70710 \\
\hline & & & 0.5 & 0.75727 & 0.39007 & 0.67750 & 0.71157 & 0.75527 & 0.38959 & 0.67950 & 0.71752 \\
\hline & & \multirow[t]{3}{*}{0.5} & 0.0 & 0.958165 & 0.403109 & 0.70020 & 0.60921 & 0.95816 & 0.40308 & 0.70939 & 0.71977 \\
\hline & & & 0.1 & 0.986898 & 0.406527 & 0.783450 & 0.614680 & 0.97432 & 0.40325 & 0.74475 & 0.72072 \\
\hline & & & 0.5 & 1.15432 & 0.43264 & 0.934562 & 0.614656 & 1.03790 & 0.40400 & 0.85815 & 0.71907 \\
\hline & & \multirow[t]{3}{*}{2.0} & 0.0 & 2.32158 & 0.39426 & 1.64207 & 0.59680 & 2.31558 & 0.40376 & 1.57329 & 0.63482 \\
\hline & & & 0.1 & 2.31601 & 0.40038 & 1.662080 & 0.59212 & 2.30204 & 0.40442 & 1.58880 & 0.63099 \\
\hline & & & 0.5 & 2.28321 & 0.41096 & 1.83220 & 0.58656 & 2.26307 & 0.40601 & 1.64517 & 0.61714 \\
\hline & \multirow{9}{*}{$\begin{array}{l}4 . \\
0\end{array}$} & \multirow[t]{3}{*}{0.2} & 0.0 & 0.627031 & 0.504676 & 0.51382 & 0.91234 & 0.62705 & 0.50459 & 0.50375 & 0.89314 \\
\hline & & & 0.1 & 0.681575 & 0.507192 & 0.55848 & 0.925677 & 0.65772 & 0.50664 & 0.54839 & 0.90091 \\
\hline & & & 0.5 & 0.859094 & 0.519451 & 0.68500 & 0.929532 & 0.76231 & 0.51242 & 0.68357 & 0.91428 \\
\hline & & \multirow[t]{3}{*}{0.5} & 0.0 & 0.993653 & 0.528672 & 0.72885 & 0.92676 & 0.99206 & 0.52979 & 0.72562 & 0.91552 \\
\hline & & & 0.1 & 1.022091 & 0.528510 & 0.76001 & 0.92753 & 1.00403 & 0.52940 & 0.75906 & 0.91694 \\
\hline & & & 0.5 & 1.08649 & 0.528002 & 0.87124 & 0.92868 & 1.05649 & 0.52858 & 0.86809 & 0.91701 \\
\hline & & \multirow[t]{3}{*}{2.0} & 0.0 & 2.41932 & 0.52779 & 1.62966 & 0.83124 & 2.41602 & 0.53103 & 1.62455 & 0.81893 \\
\hline & & & 0.1 & 2.40021 & 0.52998 & 1.64827 & 0.83099 & 2.39539 & 0.53201 & 1.63682 & 0.81623 \\
\hline & & & 0.5 & 2.29886 & 0.53124 & 1.69368 & 0.82122 & 2.28866 & 0.53467 & 1.68361 & 0.80587 \\
\hline
\end{tabular}


Table 2: Results for $f^{\prime \prime}(0)$ and $-\theta^{\prime}(0)$ for $\operatorname{Pr}=0.71, \mathrm{Ec}=0.1, \varepsilon_{0}=0.4$ for uniform permeability (UP) and variable permeability (VP).

\begin{tabular}{|c|c|c|c|c|c|c|c|c|}
\hline \multirow{2}{*}{ M } & \multirow{2}{*}{$\sigma^{*}$} & \multirow{2}{*}{$\alpha^{*} / \sigma \operatorname{Re}$} & \multirow[t]{2}{*}{$G r / \operatorname{Re}^{2}$} & \multirow{2}{*}{$\beta^{*}$} & \multicolumn{2}{|l|}{ UP } & \multicolumn{2}{|l|}{ VP } \\
\hline & & & & & $f^{\prime \prime}(0)$ & $-\theta^{\prime}(0)$ & $f^{\prime \prime}(0)$ & $-\theta^{\prime}(0)$ \\
\hline \multirow[t]{16}{*}{0.0} & \multirow[t]{12}{*}{2.0} & \multirow[t]{11}{*}{0.1} & \multirow[t]{4}{*}{0.0} & 0.0 & 0.456893 & 0.300826 & 0.467886 & 0.396778 \\
\hline & & & & 0.1 & 0.570024 & 0.326784 & 0.573214 & 0.399978 \\
\hline & & & & 0.5 & 0.994532 & 0.456781 & 0.984919 & 0.524678 \\
\hline & & & & 0.9 & 1.304657 & 0.700567 & 1.294623 & 0.800321 \\
\hline & & & \multirow[t]{4}{*}{0.1} & 0.0 & 0.559087 & 0.299945 & 0.553608 & 0.386262 \\
\hline & & & & 0.1 & 0.679265 & 0.356782 & 0.682864 & 0.414960 \\
\hline & & & & 0.5 & 1.159872 & 0.713457 & 1.135678 & 0.800032 \\
\hline & & & & 0.9 & 1.364879 & 0.800267 & 1.354657 & 0.834567 \\
\hline & & & \multirow[t]{2}{*}{0.2} & 0.0 & 0.668432 & 0.302458 & 0.685034 & 0.396120 \\
\hline & & & & 0.1 & 0.784567 & 0.306892 & 0.793051 & 0.399673 \\
\hline & & & 2.0 & 0.1 & 2.264556 & 0.425674 & 2.262345 & 0.419082 \\
\hline & & 0.5 & 0.2 & 0.0 & 0.924356 & 0.398543 & 0.926342 & 0.437865 \\
\hline & \multirow[t]{4}{*}{4.0} & \multirow[t]{4}{*}{0.1} & \multirow[t]{2}{*}{0.0} & 0.1 & 0.548945 & 0.480678 & 0.569842 & 0.548623 \\
\hline & & & & 0.5 & 0.948720 & 0.700467 & 0.967454 & 0.741265 \\
\hline & & & \multirow[t]{2}{*}{0.2} & 0.1 & 0.665684 & 0.523678 & 0.673454 & 0.554929 \\
\hline & & & & 0.5 & 1.003451 & 0.624563 & 1.024656 & 0.654667 \\
\hline \multirow[t]{7}{*}{1.0} & \multirow[t]{5}{*}{2.0} & \multirow[t]{4}{*}{0.1} & \multirow[t]{3}{*}{0.2} & 0.0 & 0.965478 & 0.356912 & 0.976456 & 0.394134 \\
\hline & & & & 0.1 & 1.231065 & 0.367823 & 1.237803 & 0.400345 \\
\hline & & & & 0.5 & 1.481910 & 0.398795 & 1.503830 & 0.426574 \\
\hline & & & 2.0 & 0.1 & 2.321546 & 0.456321 & 2.331602 & 0.445354 \\
\hline & & 0.5 & 2.0 & 0.0 & 0.999854 & 0.423456 & 1.003782 & 0.413678 \\
\hline & \multirow[t]{2}{*}{4.0} & \multirow[t]{2}{*}{0.1} & \multirow[t]{2}{*}{0.2} & 0.1 & 0.775892 & 0.625489 & 0.786724 & 0.642363 \\
\hline & & & & 0.5 & 1.494300 & 0.536123 & 1.504808 & 0.544071 \\
\hline \multirow[t]{7}{*}{5.0} & \multirow[t]{5}{*}{2.0} & \multirow[t]{4}{*}{0.1} & \multirow[t]{3}{*}{0.2} & 0.0 & 1.358431 & 0.415678 & 1.365432 & 0.435216 \\
\hline & & & & 0.1 & 1.454890 & 0.427856 & 1.465390 & 0.446782 \\
\hline & & & & 0.5 & 1.668054 & 0.448961 & 1.689042 & 0.458653 \\
\hline & & & 2.0 & 0.1 & 2.435765 & 0.426718 & 2.446790 & 0.435673 \\
\hline & & 0.5 & 2.0 & 0.0 & 1.504789 & 0.419836 & 1.524789 & 0.423478 \\
\hline & 4.0 & 0.1 & 0.2 & 0.1 & 1.327643 & 0.638956 & 1.345626 & 0.642345 \\
\hline & & & & 0.5 & 1.765902 & 0.538713 & 1.856409 & 0.547658 \\
\hline 10.0 & 2.0 & 0.1 & 0.2 & 0.0 & 1.534662 & 0.467843 & 1.756765 & 0.556765 \\
\hline
\end{tabular}

Fig.2. shows the nusselt number variations with different prandtl numbers and $\sigma^{*}$ for both UP and VP cases, lower the prandtl no. with the increase $\sigma^{*}$ and $\mathrm{Gr} / \mathrm{Re}^{2}$ the heat transfer is slightly increasing and decreasing in low range, higher the prandtl no. with the increase of $\sigma^{*}$ and $\mathrm{Gr} / \mathrm{Re}^{2}$ the heat transfer is slightly increasing and then decreasing in high range. For a very small value of $\mathrm{M}$ the results are comparable with the existing Mohammedein and El-shaer.

Fig.3. shows the velocity distribution for various values of magnetic field parameter $M$ for variable permeability case. Here the force due to magnetic field acts as an accelerating force and reduces the frictional resistance which leads to velocity profile increase as we increase the value of $\mathrm{M}$ within the boundary layer and the boundary layer increases with decrease in the value of $\mathrm{M}$.

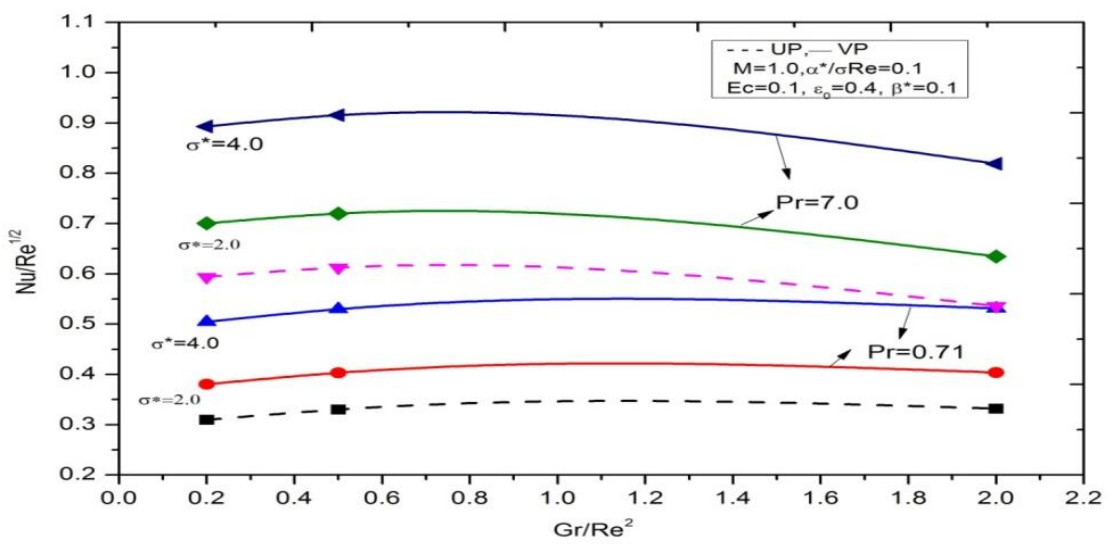

Fig.2.Nusselt number for various $\mathrm{Gr} / \mathrm{Re}^{2}, \sigma^{*}$ and $\operatorname{Pr}$ for UP and VP

Fig.4. shows the variation of velocity distribution for various values of second-order resistance (Forchheimer drag) for variable permeability case. Due to the effect of more resistance by the porous medium to the fluid flow we 
observe that, increase in the value of inertial parameter $\beta^{*}$ leads to increase in the velocity profile within the boundary layer. We also observe that the boundary layer thickness also decreases with an increase in the value of inertial parameter.

Fig.5. shows the variation of velocity distribution for different values of the parameter $\mathrm{Gr} / \mathrm{Re}^{2}$ for both the cases of UP and VP. In determining the combined flow the magnitude of $\mathrm{Gr} / \mathrm{Re}^{2}$ shows the relative importance. Here we see that increase in the value of $\mathrm{Gr} / \mathrm{Re}^{2}$ increases the velocity distribution for both the cases and it is very significant for higher value of $\mathrm{Gr} / \mathrm{Re}^{2}$ in the boundary layer. For some particular value of $\mathrm{Gr} / \mathrm{Re}^{2}$ the velocity profile is found to be less for VP as compared to UP. Further, it is clear that the boundary layer decreases with increase in the value of $\mathrm{Gr} / \mathrm{Re}^{2}$. The free convection currents obtained during the process of cooling of plate induce the mean velocity to increase. Thus increase in the values of $\mathrm{Gr} / \mathrm{Re}^{2}$ have the tendency to increase the buoyancy effects causing more induced flow along the plate in the vertical direction reflected by the increase in the fluid velocity.

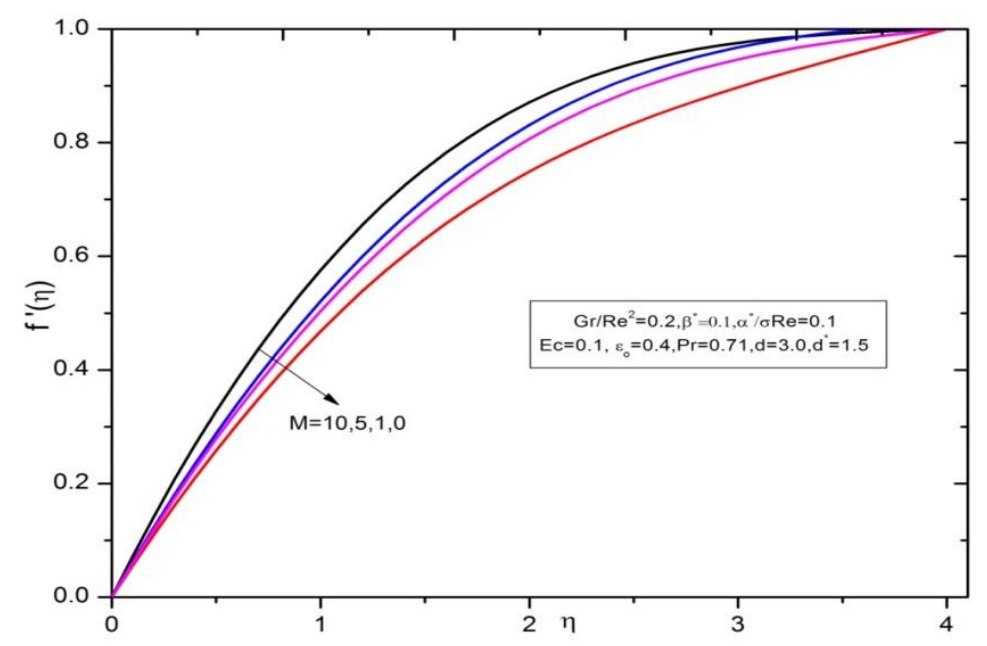

Fig. 3. Velocity profiles for different values of $\mathrm{M}$ for VP

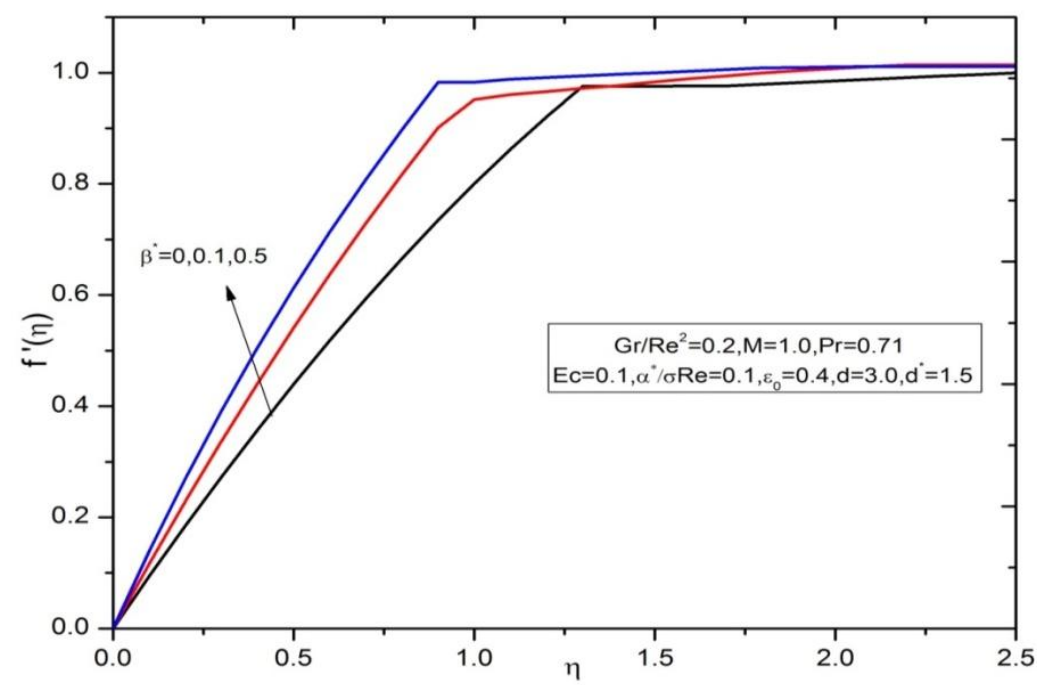

Fig.4. Velocity profiles for different values of $\beta^{*}$ for VP

Fig.6. shows the variations of velocity profiles for various values of $\alpha^{*} / \sigma$ Refor both UP and VP. It is clearly seen that the velocity profile increases with an increase in $\alpha^{*} / \sigma$ Re. The velocity profiles for VP are less compared to UP which can be clearly observed for higher values of $\alpha^{*} / \sigma$ Re.

Fig.7.shows the velocity profiles for different values Prandtl numbers for both VP and UP case. It is observed that the velocity profiles decreases as the Prandtl number increases which is very significant in the boundary layer, and also the boundary layer decreases with decrease in the value of Pr. 
Fig.8. shows the temperature profiles for various values of magnetic parameter $M$ for VP, it is seen that the temperature profile decreases for all values of magnetic parameter $\mathrm{M}$ within the boundary layer. In the absence of Magnetic parameter the results are compared with the existing results.

Fig.9. shows the temperature distribution for various values of second order resistance $\beta^{*}$ for both UP and VP cases. Here the temperature profile decreases for $\beta^{*}=0.0$ within the boundary layer and also for higher values of $\beta^{*}$, temperature continuously decreases, which means rate of cooling is much faster for higher values of second order resistance in both the cases.

Fig.10. shows the temperature profiles for various values of $G r / \mathrm{Re}^{2}$ for both UP and VP cases. It is observed that increase in the value of $\mathrm{Gr} / \mathrm{Re}^{2}$ decreases the temperature distribution in both the cases.

Fig.11. is the plot of variations of temperature profiles for various values of $\alpha^{*} / \sigma \operatorname{Re}$ for both UP and VP. Here it is noted that the temperature profiles increases with decreasing the value of $\alpha^{*} / \sigma \operatorname{Re}$ within the boundary layer for both UP and VP cases. It is observed that temperature in the boundary layer is less for VP case for all values of $\alpha * / \sigma \operatorname{Re}$. This fact shows that the rate of cooling is much faster in the case of UP for all values of $\alpha^{*} / \sigma \operatorname{Re}$.

Fig.12. shows the variation of temperature profiles for various values of $\sigma^{*}$ for UP and VP cases. From this figure it is evident that the temperature profile increases by increasing the values of $\sigma^{*}$ within the boundary layer for both UP and VP cases. The effect of $\sigma^{*}$ is to increase the temperature in the boundary layer and temperature for UP is always less compared to VP case.

Fig.13. shows the temperature profiles for various values of Prandtl numbers for VP case. The temperature profiles show a typical smooth decreasing pattern for lower Pr whereas, for higher values of Pr, the temperature continuously decreases at a steeper rate in the flow region and the boundary layer decreases.

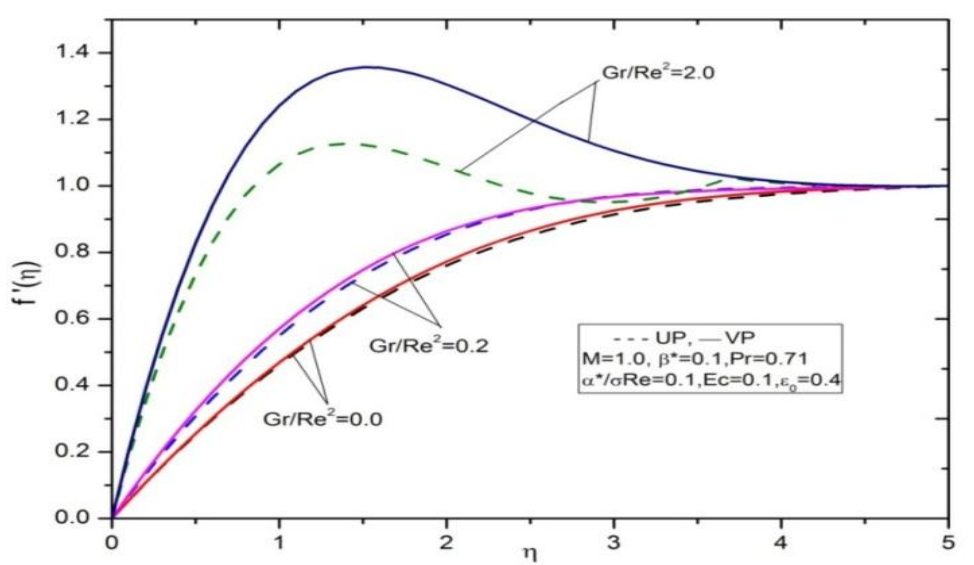

Fig.5. Velocity profiles for different values of $\mathrm{Gr} / \mathrm{Re}^{2}$ for UP and VP

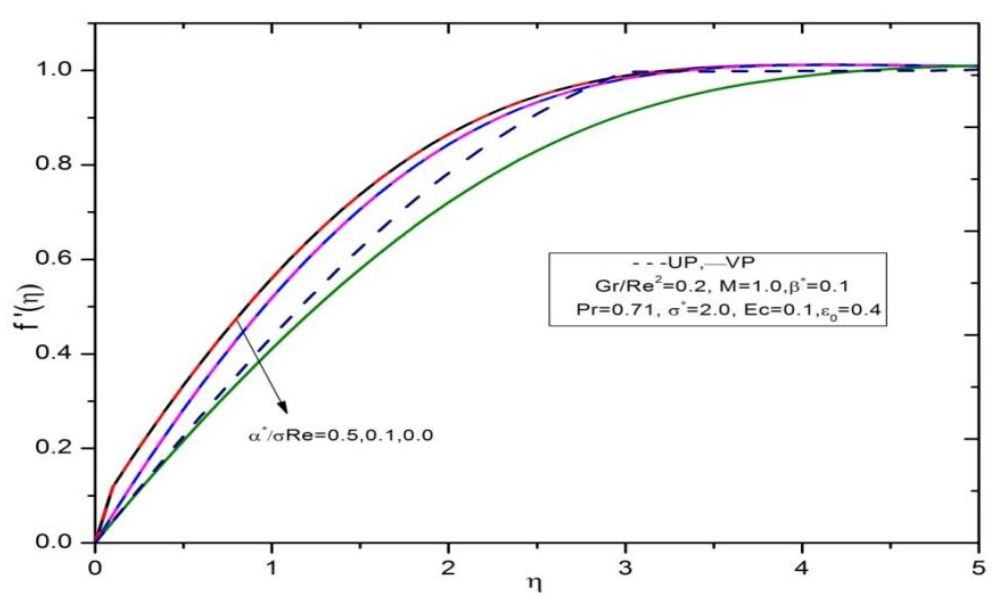

Fig.6.Velocity profiles for different values of $\alpha^{*} / \sigma \operatorname{Re}$ for UP and VP 


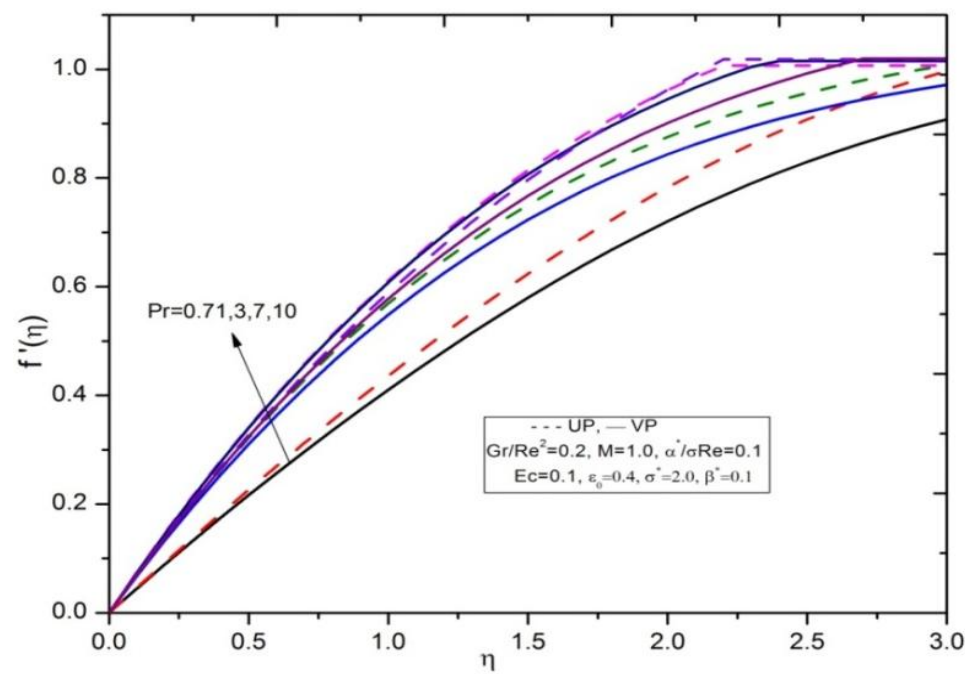

Fig.7. Velocity profiles for different values of Pr for VP

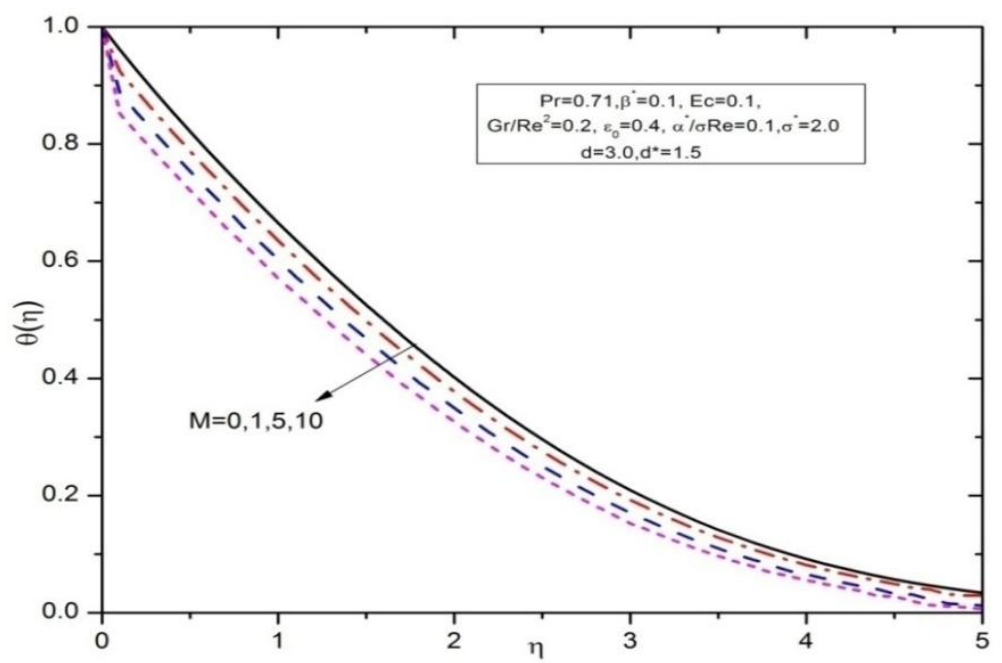

Fig.8. Temperature profiles for various values of $\mathrm{M}$ for VP

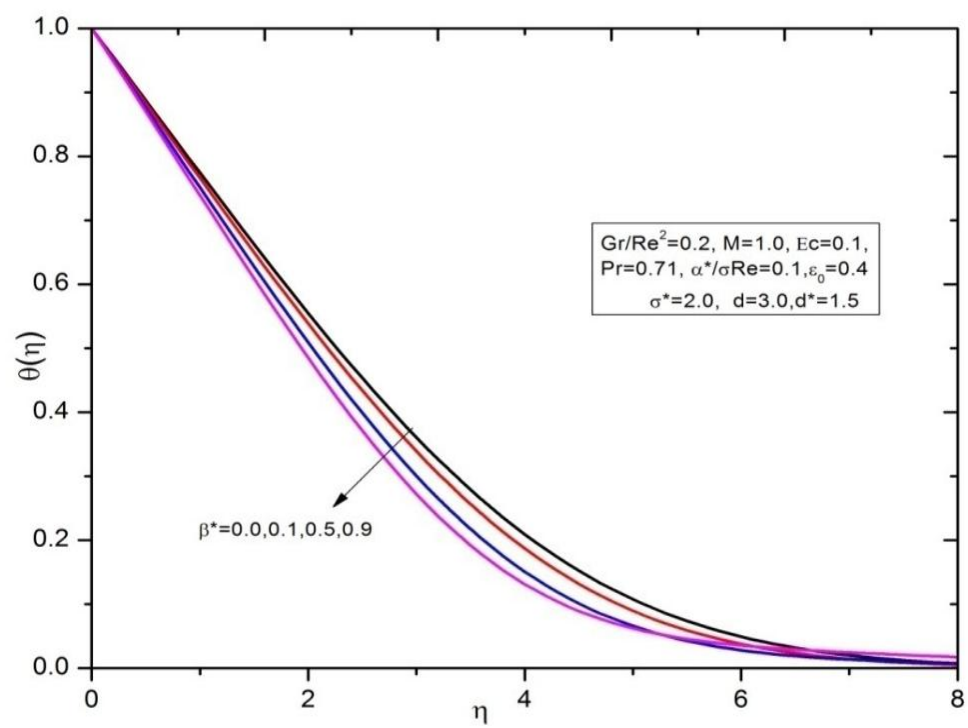

Fig. 9. Temperatur profiles for various values of $\beta^{*}$ for VP 


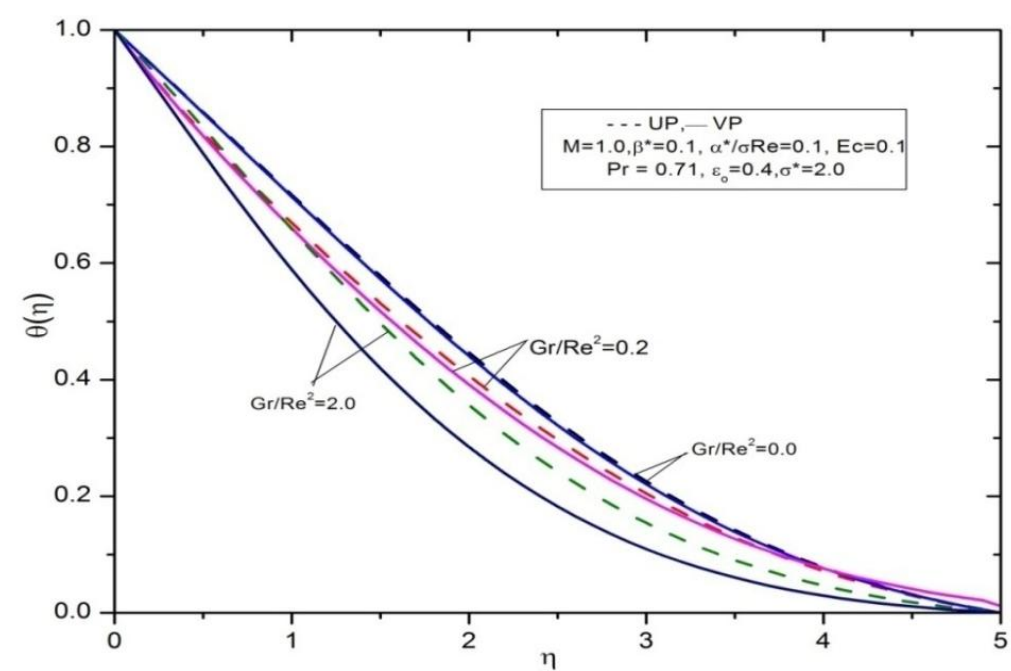

Fig.10. Temperature profiles for various values of $\mathrm{Gr} / \mathrm{Re}^{2}$ for UP and VP

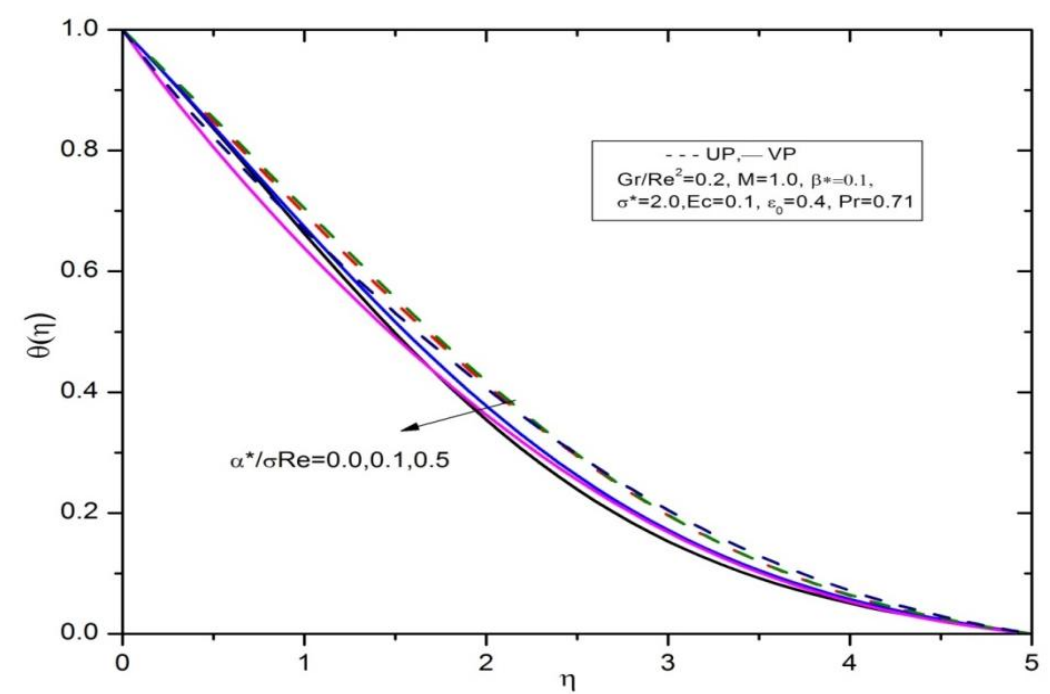

Fig.11. temperatures profiles for various values of $\alpha^{*} / \sigma \operatorname{Re}$ for both UP and VP cases

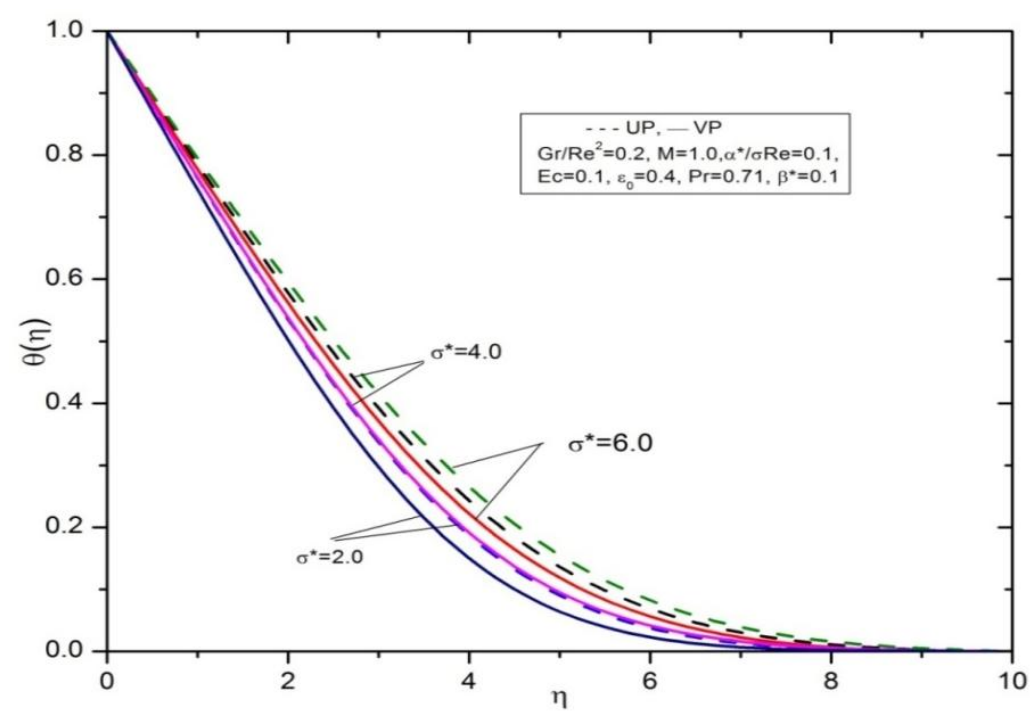

Fig. 12. Temperature profiles for various values of $\sigma^{*}$ for UP and VP 


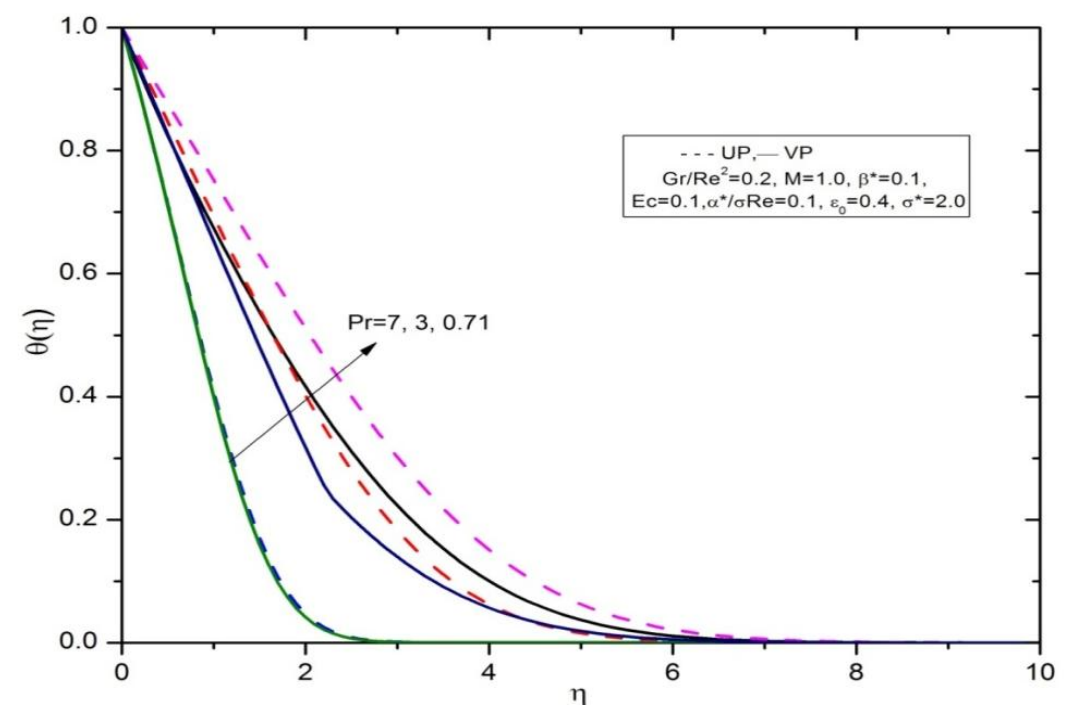

Fig. 13. Temperature profiles for various values of Pr for VP

\section{Conclusions}

In this study, a numerical approach is made to study the effect of variable fluid properties like variable permeability, porosity and thermal conductivity on Mixed convection from a vertical heated plate embedded in a electrically conducting fluid saturated sparsely packed porous medium with an applied magnetic field normal to the plate. The boundary layer flow in the porous medium is governed by Brinkman - Forchheimer extended Darcy model. The conservation equations governing the problem are reduced to a system of non-linear ordinary differential eequations by using similarity transformations which are highly coupled. These equations are then solved numerically by Runge - Kutta method with shooting technique. The computed results which are tabulated are shown graphically to illustrate the flow and heat transfer characteristics and also their dependence on various physical parameters. The following conclusions are drawn:

1. The effect of magnetic field $M$ is to increase the velocity distribution significantly and there is decrease in the temperature distribution in the thermal boundary layer for variable permeability case.

2. As the values of inertial parameter $\beta^{*}$ increases, there is a decrease in the boundary layer thickness for variable permeability case in case of both velocity and temperature profiles.

3. Increase in the value of buoyancy parameter $\mathrm{Gr} / \mathrm{Re}^{2}$ leads to increase in the velocity closer to the vertical plate with peak value increase for higher values of $G r / \mathrm{Re}^{2}$ for both the cases of UP and VP, also to decrease the temperature profile in the boundary layer for both the cases of VP and UP. We observe that temperature profiles for VP are always less compared to UP.

4. The velocity profile increases with an increase in $\alpha * / \sigma$ Re whereas the temperature profiles increases with decreasing the value $\alpha * / \sigma \operatorname{Re}$. The velocity and temperature profiles for VP are less compared to UP.

5. The effect of increasing the values of $\sigma *$ increases temperature, whereas the reverse trend is seen by increasing Pr in the thermal boundary layer for both UP and VP cases.

\section{Acknowledgements}

The authors are grateful to the Research Centre M S Ramaiah Institute of Technology, Atria Institute of Technology, Vivekananda Institute of Technology, for all the support and also the financial support from VTU research scheme project.

\section{References}

[1] J H Merkin The effects of buoyancy forces on the boundary layer flow over a semi infinite vertical plate in a uniform free stream, $J$ Fluid Mech., Vol.35, 1969, 439-450.

[2] P Ranganathann, R Viskanta, Mixed convection boundary layer flow along a vertical surface in a porous medium. Num Heat Transfr 7 , 1984, 305-317.

[3] J.C. Hsieh, T.S. Chen, B.F.Armaly, Non-similarity solutions for mixed convection from vertical surfaces in porous medium with variable surface temperature or heat flux, Int.J.Heat Mass Transfer, vol.38,No.4,1993,1485-1493.

[4] B. Saltzmann, The General Circulation as a problem in Thermal Convection: A Collection of Classical and Modern Theoretical Papers, Dept. of Meteorology, Massachusetts Institute of Technology (1958)

[5] T.K.Aldoss, M.A. Al-Nimr, M.A.Jarrah, B.J.Al-shaer, Magnetohydrodynamic Mixed Convection from a vertical plate embedded in a porous medium, Numerical Heat Transfer: An International Journal of Computation and Methodology. Vol.28, Issue 5. 1995,.635-645

[6] D.A.Nield and A. Bejan Convection in porous media.(Springer verlag 1999). 
[7] E.M.A. Elabashbeshy, Heat and mass transfer along a vertical plate with variable temperature and concentration in the presence of magnetic field. Int.J. Eng. Sci., 34, 1997,515-522.

[8] C.E.Schwartz and J.M.Smith, Flow distribution in packed beds. Ind.Eng.Chem., vol.45, 1953,1209-1218.

[9] R.F. Benanati and C.B.Brosilow, Void fraction distribution in beds of spheres.-AIChe J., vol.8, 1962,359-361

[10] B.C. Chandrasekhara, P.M.S.Namboodiri, A R.Hanumanthappa, Similarity solutions of buoyancy induced flows in a saturated porous medium adjacent to impermeable horizontal surfaces. Warme Stoffubertrag, 1984;18:17-23.

[11] B.C. Chandrasekhara and P.M.S.Namboodiri, Influence of variable permeability on combined vertical surfaces in porous medium.International Journal of Heat Mass Transfer, vol.28, 1985, 199-206.

[12] A.A. Mohammadein and N.A. El-Shaer, Influence of variable permeability on combined free and forced convection flow past a semi infinite vertical plate in a saturated porous medium.- Heat Mass Transfer, vol.40, 2004, 341-346.

[13] V. Sri Hari Babu and G.V.Ramana Reddy, Mass transfer effects on MHD mixed convective flow from a vertical surface with ohmic heating and viscous dissipation. Adv. Appl.Sci.Res., 2(4), 2011,138-146.

[14] Mahinder Singh, C.B. Mehta and Sanjeev Gangta, Thermosolutal Convection in Compressible, Rotating Couple-Stress Fluid in The Presence of Magnetic field. Adv. Appl. Sci. Res., 3(6), 2012,3459-3468.

[15] D. Pal, I S Shivakumara, Mixed Convection heat transfer from a vertical heated plate embedded in a sparsely packed porous medium. Int J Appl Mech Eng, 11,2006,929-39.

[16] Dulal Pal, Magnetohydrodynamic non-Darcy mixed convection heat transfer from a vertical heated plate embedded in a porous medium with variable porosity,- Commun Nonlinear Sci Numer Simulat. Vol.15,2010, 3974-3987.

[17] Dulal Pal, Mixed convection heat transfer in the boundary layers on an exponentially stretching surface with magnetic field, Applied Mathematics and Computation 217,2010, 2356-2369

[18] N. Nalinakshi, P.A.Dinesh, I.S. Shivakumara, D.V.Chandrashekar, Numerical Solution for Mixed Convection Heat Transfer from a Vertical Heated Plate Embedded in a Sparsely Packed Porous Medium, J Mapana, Vol.10, No.2, 2011, $37-53$.

[19] Vajravelu K., Viscous flow over a nonlinear stretching sheet. Applied Math Computation,vol.124,2001,281-288. 